\title{
Association of Rush Pin and Intramedular Pin Techniques for the Stabilization of Salter Harris Type I Fracture in Rabbit (Oryctolagus cuniculus)
}

\author{
Fernando Yoiti Kitamura Kawamoto', Lívia Perles², Levi Oliveira dos Santos', Giulia Carneiro Simionato ${ }^{1}$, \\ Luis Gustavo Gosuen Gonçalves Dias', Bruno Watanabe Minto' ${ }^{1}$ \& Karin Werther ${ }^{2}$
}

\begin{abstract}
Background: In domestic rabbits, fractures are usually the result of household accidents. Fractures of the distal femoral physis are frequently observed in animals with immature skeletons and may cause future orthopedic problems. With this type of fracture, early reduction and stabilization are necessary to prevent additional damage to the physis and to preserve the growth potential of the bone. This report aims to describe the clinical and radiographic findings, as well as the surgical method used that combined Rush pins with an intramedullary pin, associated with a Salter Harris type I fracture of the right distal femur in a rabbit.

Case: A 4-month male domestic rabbit (Oryctolagus cuniculus) weighing $1.2 \mathrm{~kg}$, was referred to the Veterinary Hospital with a right pelvic limb lameness after an episode of trauma. Orthopedic examination revealed swelling, instability, crepitation, and increased pain sensitivity in the distal aspect of the right femur. Following physical and radiographic examination, a Salter Harris type I fracture of the right distal femur was diagnosed. The patient was premedicated with a combination of xylazine ( $2 \mathrm{mg} / \mathrm{kg} \mathrm{IM})$ and ketamine $(10 \mathrm{mg} / \mathrm{kg} \mathrm{IM})$, which allowed placement of an IV catheter for the administration of fluids and intubation using an endotracheal tube with an internal diameter of $2 \mathrm{~mm}$. Anesthesia was maintained with isoflurane, and an epidural was performed with a combination of lidocaine $(2 \mathrm{mg} / \mathrm{kg})$ and bupivacaine $(0.75 \mathrm{mg} / \mathrm{kg})$. Osteosynthesis was performed with two Rush pins and an intramedullary pin. Radiographic examination 110 and 330 days after the surgical procedure showed good alignment of the bone and adequate healing of the fracture. The combination of techniques used in this case report proved to be effective, resulting in functional recovery of the limb and rapid bone healing. Discussion: Preoperative planning for orthopedic surgery in rabbits is different from that of dogs and cats, due to the particularities of the species. Familiarity with the regional anatomy, patient preparation, and appropriate instrumentation are necessary when contemplating osteosynthesis in a rabbit. Salter Harris fractures affect young animals, where the physis is considered an area of fragility in the bone. Surgical planning should take into account the function of the growth plates. It is recommended that implants passing through the physis do so perpendicularly, since angulation greater than $45^{\circ}$ may predispose the bone to premature closure of the growth plate. Another important consideration involves the choice of implants, since the use of trocar-tipped pins facilitates their precise placement in the bone. The use of threaded pins should be avoided due to their weakness at the thread-shaft interface, and the risk of impaired longitudinal bone growth and the difficulty of removal if necessary. Steinman pins and Kirschner wires can be used to stabilize a variety of different fractures. In Salter-Harris type I and II fractures, the use of pins neutralizes bending forces but not rotational or compressive forces. In contrast, Rush pinning and cross-pinning techniques are effective in neutralizing the forces acting on the physis, and are frequently used for the fixation of fractures in this region. Complications, often associated with poor reduction and alignment, can result in varus or valgus deviation and are associated with a high risk of implant failure or migration, malunion, and patellar luxation. It was concluded that the combination of Rush pins and an intramedullary pin resulted in adequate stabilization of the Salter Harris type I fracture of the distal femur in this rabbit.
\end{abstract}

Keywords: Orthopedic implants, osteosynthesis, trauma, bone repairing, lagomorph. 


\section{INTRODUCTION}

In domestic rabbits, fractures are usually the result of household accidents such as an owner stepping on or a door closing on the animal, a fall, being crushed by a piece of furniture, vehicular trauma, or a dog or cat bite $[6,12]$.

Fractures of the distal femoral physis are frequently observed in animals with immature skeletons [1] and may cause future orthopedic problems due to limb shortening, angular deformity, and/or joint incongruity [15]. With this type of fracture, early reduction and stabilization are necessary to prevent additional damage to the physis and to preserve the growth potential of the bone [3].

Fracture stabilization can be performed via an open or closed approach $[3,8,13]$. Particularly in cases with chronic fractures, muscle contraction and periarticular fibrosis limit the applicability of closed methods because of difficulty in aligning the fracture fragments. Although some stability is provided by anatomical reduction, the strength of the physeal interdigitations alone is not sufficient to counteract shear and rotational forces. There are several proven techniques that can be used for fracture stabilization $[3,8]$.

The use of intramedullary pins in rabbits is common. These implants are affordable and simple to apply. Most techniques utilizing this type of implant also require at least two Kirschner wires or small diameter Steinmann pins, preferably applied in normograde fashion, to achieve adequate stability [6].

This report aims to describe the clinical and radiographic findings, as well as the surgical method used that combined Rush pins with an intramedullary pin, associated with a Salter Harris type I fracture of the right distal femur in a rabbit.

\section{CASE}

A 4-month male domestic rabbit (Oryctolagus cuniculus) weighing $1.2 \mathrm{~kg}$, was referred to our hospital for evaluation of a right pelvic limb lameness after an episode of trauma. Aside from the lameness, no abnormalities were observed on physical examination. Orthopedic examination revealed instability and crepitation of the right femoral-tibial-patellar articulation. Radiographs revealed a Salter Harris type I fracture of the right distal femur (Figure 1A). Osteosynthesis was performed combining Rush pinning with an intramedullary pin technique.
The patient was premedicated with a combination of $2 \mathrm{mg} \mathrm{kg}$ xylazine $\left(\text { Xilazin }^{\circledR}\right)^{1}$ and $10 \mathrm{mg} \mathrm{kg}$ ketamine $\left(\operatorname{Cetamin}^{\circledR}\right)^{1}$, given intramuscularly, which allowed placement of an intravenous catheter for the administration of fluids and intubation using an endotracheal tube with an internal diameter of $2 \mathrm{~mm}$. Anesthesia was maintained with isoflurane, and an epidural was performed with a combination of $2 \mathrm{mg} \mathrm{kg}$ lidocaine $\left(\text { Anestt }^{\circledR}\right)^{1}$ and $0.75 \mathrm{mg} \mathrm{kg}$ bupivacaine $\left(\text { Neocaína }{ }^{\circledR}\right)^{2}$.

The hair was clipped from the right pelvic limb, which was aseptically prepared using chlorhexidine $2 \%$ and isopropyl alcohol $70 \%$. The patient was then positioned in left lateral recumbency. A lateral parapatellar incision was made, exposing the knee joint capsule, which was later incised to expose the joint. A 1.5-mm Kirschner wire, used as an intramedullary pin, was placed in a normograde fashion exiting the distal fracture fragment cranial to the origin of the cranial cruciate ligament, thereby reducing the fracture. Two 1-mm Kirschner wires were then placed, again in a normograde fashion, from the lateral and medial femoral condyles, respectively, as Rush pins. After the stability of the repair was verified, the exposed distal portions of the pins were trimmed and smoothed. Finally, the subcutaneous tissue and skin were closed routinely using monofilament absorbable suture. Postoperatively the patient was treated with $5 \mathrm{mg} \mathrm{kg}$ enrofloxacin $\left(\text { Chemitril }^{\circledR}\right)^{3}$ subcutaneously BID for 7 days, $0.1 \mathrm{mg}$ $\mathrm{kg}$ meloxicam $\left(\text { Meloxicam }^{\circledR}\right)^{4}$ subcutaneously SID for 3 days, and $5 \mathrm{mg} \mathrm{kg}$ tramadol chloride $\left(\text { Tramadon }^{\circledR}\right)^{2}$ intramuscularly BID for 3 days.

Immediate postoperative radiographs showed correct positioning of the implants, with adequate reduction and alignment of the fracture fragments (Figure 1B and 1C).

Ten days postoperatively, the skin sutures were removed. The animal presented functional support of the injured limb at that time. Follow-up radiographs were performed 110 days postoperatively (Figure 1D). Complete healing of the bone was observed, and clinically presented with normal limb function. The same observations were made 330 days postoperatively (Figure 1E and 1F).

\section{DISCUSSION}

Preoperative planning for orthopedic surgery in rabbits is different from that of dogs and cats, due to the particularities of the species [6]. Familiarity 


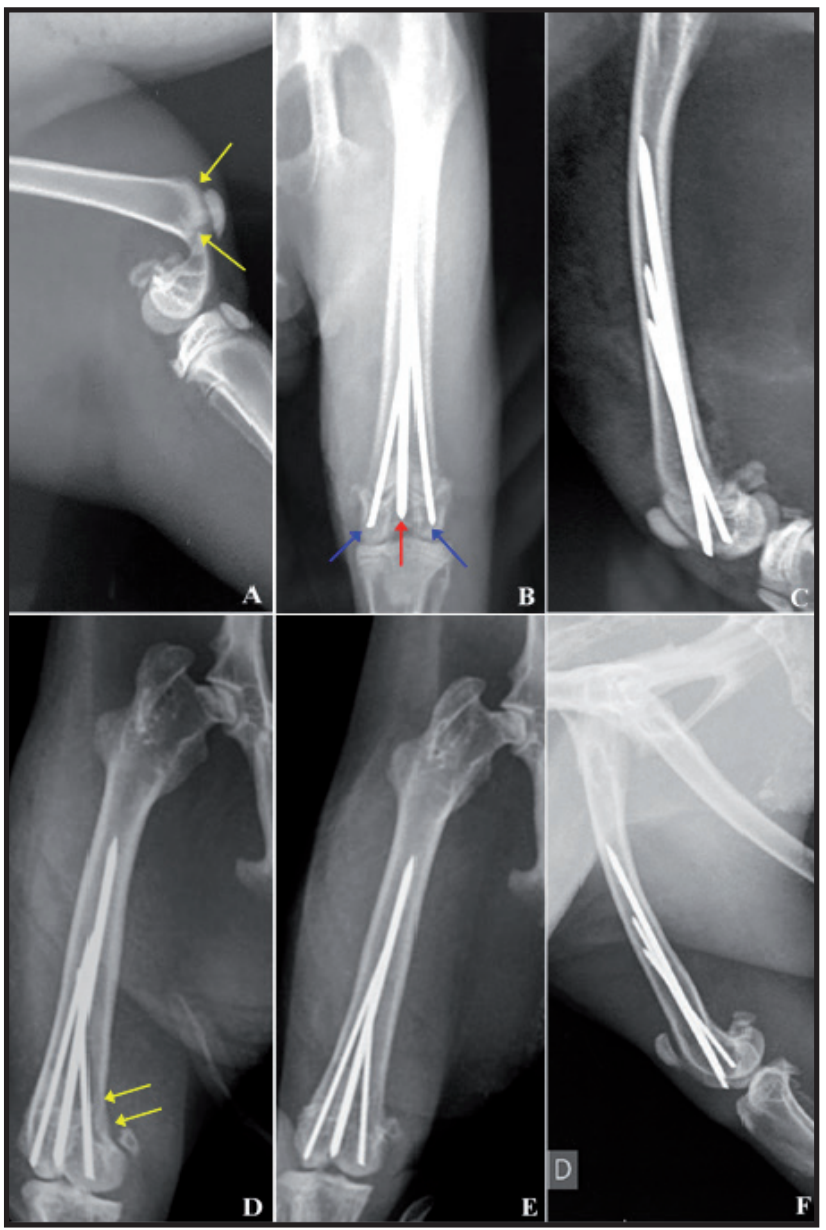

Figure 1. Radiographic images (craniocaudal and mediolateral projections) of the right femur of a rabbit (Oryctolagus cuniculus). A- Preoperative image showing a right distal femoral fracture (Salter Harris type I - yellow arrows). $\mathrm{B}$ and C-Immediate postoperative images. Osteosynthesis was performed with an intramedullary pin (red arrow) and two Kirschner wires used as Rush pins (blue arrows). Good bone alignment is observed, with fragment apposition and appropriately sized implants. D- Radiographic image obtained 110 days postoperatively, evidencing fracture consolidation (yellow arrows). E and F- Radiographic images obtained 330 days postoperatively.

with the regional anatomy, patient preparation, and appropriate instrumentation are necessary when contemplating osteosynthesis in a rabbit. In addition, prior knowledge of the physiology, response to nociception, and predisposition of these animals to gastrointestinal stasis following surgical procedures is important $[2,12]$.

Rabbits undergoing surgical procedures should be monitored for defecation and water and food consumption [5,9], which must be normalized within 24-48 $\mathrm{h}$ postoperatively [12]. In this case, the patient's appetite returned after recovering from anesthesia. No signs of gastrointestinal stasis were observed during the postoperative period.

Although sedation or general anesthesia is often recommended for radiographic examination in rabbits $[6,8]$, in this case, it was not required, and the patient was appropriately positioned using physical restraint alone.
Salter Harris fractures affect young animals, where the physis is considered an area of fragility in the bone $[4,10,14]$. According to Pearce et al. [11], the epiphyseal plates in rabbits close at approximately six months of age. This correlates with the findings in this patient who had, at four months of age, open growth plates visible on radiographic images. Surgical planning should take into account the function of the growth plates. It is recommended that implants passing through the physis do so perpendicularly, since angulation greater than $45^{\circ}$ may predispose the bone to premature closure of the growth plate $[1,4]$. Another important consideration involves the choice of implants, since the use of trocar-tipped pins facilitates their precise placement in the bone. The use of threaded pins should be avoided due to their weakness at the thread-shaft interface, and the risk of impaired longitudinal bone growth and the difficulty of removal if necessary [8]. Taking into account all applicable orthopedic principles, a trocar-tipped Steinman pin was chosen in this case, and no significant interference with limb growth was observed. Steinman pins and Kirschner wires can be used to stabilize a variety of different fractures. In Salter-Harris type I and II fractures, the use of pins neutralizes bending forces but not rotational or compressive forces. In contrast, Rush pinning and cross-pinning techniques are effective in neutralizing the forces acting on the physis, and are frequently used for the fixation of fractures in this region $[1,3,10]$. A combination of the two techniques conferred greater stability to the repair in this case, resulting in an early return to function and effective healing of the fracture.

Kim et al. [8] recommended the insertion of pins percutaneously to avoid iatrogenic trauma to important structures such as the joint capsule and physis, reduce postoperative pain, and accelerate bone healing. However, in this case, an open approach was used for reduction of the fracture and visualization of the implants, mainly due to the absence of intraoperative fluoroscopy. In addition, the small size of the fragments made it difficult to identify and manipulate them via a closed approach.

In general, physeal fractures result in premature closure of the growth plates and subsequent angular limb deformities [4]. According to the literature, this is observed in up to $83 \%$ of the cases and seems to be related more to the trauma itself than to the stabilization technique chosen [3]. In the radiographic examination performed 110 days postoperatively in this case, no disparity was observed in the growth of the two limbs, nor was any 
angularity observed in the injured limb. Complications often associated with poor reduction and alignment, can result in varus or valgus deviation and are associated with a high risk of implant failure or migration, malunion, and patellar luxation $[3,10]$. To date, no complications have been reported affecting the injured limb in this case.

Rabbits have a high propensity for the development of excessive granulation tissue in response to the presence of foreign material, and thus, the choice of suture material is an important consideration in rabbit surgery [5]. To prevent potential complications, a monofilament absorbent material (e.g., polydioxanone) is recommended for internal sutures. External sutures, although also preferably monofilament, should be nonabsorbable. Nylon is the most commonly used skin suture in rabbit surgery [12]. Some patients might remove external sutures and cause infection of the surgical site. Since the use of Elizabethan collars is challenging in these patients [6], the placement of intradermal sutures is often recom- mended. Even with the use of simple interrupted sutures in the skin and the absence of an Elizabethan collar, no surgical site complications were noted in this case.

It was concluded that the combination of Rush pins and an intramedullary pin resulted in adequate stabilization of the Salter Harris type I fracture of the distal femur in this rabbit. Bone consolidation and an early return to function, without any compromise to bone growth or development of any angular limb deformities, were noted in this case.

\section{MANUFACTURERS}

${ }^{1}$ Syntec. Santana de Parnaíba, SP, Brazil.

${ }^{2}$ Laboratório Cristália. São Paulo, SP, Brazil.

${ }^{3}$ Chemitec Agro Veterinária. São Paulo, SP, Brazil.

${ }^{4}$ Ouro Fino Pet Saúde Animal. Osasco, SP, Brazil.

Declaration of interest. The authors report no conflicts of interest. The authors alone are responsible for the content and writing of the paper.

\section{REFERENCES}

1 Beale B. 2004. Orthopedic clinical techniques femur fracture repair. Clinical Techniques in Small Animal Practice. 19(3): 134-50.

2 Donnelly T.M. 1997. Basic anatomy, physiology, and husbandry. In: Hillyer H.V. \& Quesenberry K.E. (Eds). Ferrets, Rabbits, and Rodents: Clinical Medicine and Surgery. Philadelphia: WB Saunders, pp.147-159.

3 Guiot L.P., Demianiuk R.M. \& Déjardin L.M. 2012. Fractures of the femur. In: Tobias K.M. \& Johnston S.A. (Eds). Veterinary Surgery Small Animal. St. Louis: Elsevier Saunders, pp.865-905.

4 Jackson L.C. \& Pacchiana P.D. 2004. Common complications of fracture repair. Veterinary Clinics of North America: Exotic Animal Practice. 19(3): 168-179.

5 Jenkins J.R. 1997. Soft tissue surgery and dental procedures. In: Hillyer H.V. \& Quesenberry K.E. (Eds). Ferrets, Rabbits, and Rodents: Clinical Medicine and Surgery. Philadelphia: WB Saunders, pp. 227-239.

6 Kapatkin A. 1997. Orthopedics in small mammals. In: Hillyer H.V. \& Quesenberry K.E. (Eds). Ferrets, Rabbits, and Rodents: Clinical Medicine and Surgery. Philadelphia: WB Saunders, pp.346-357.

7 Kelleher S. 2000. Wound and abscess management in rabbits. Exotic DVM. (2.3): 49-51.

8 Kim S.E., Hudson C.C. \& Pozzi A. 2012. Percutaneous pinning for fracture repair in dogs and cats. Veterinary Clinics of North America: Exotic Animal Practice. 42(5): 963-974.

9 Krempels D., Cotter M. \& Stanzione G. 2000. Ileus in domestic rabbits. Exotic DVM. (2.4): 19-21.

10 McLaughlin R.M. 2001. Hind limb lameness in the young patient. The Veterinary Clinics of North America: Small Animal Practice. 31(1): 101-123.

11 Pearce A., Richards R.G., Milz S., Schneider E. \& Pearce S.G. 2007. Animal models for implant biomaterial research in bone: a review. European Cells \& Materials Journal. 13: 1-10.

12 Rich G.A. 2002. Rabbit orthopedic surgery. Veterinary Clinics of North America: Exotic Animal Practice. 5(1): 157-168.

13 Sasai H., Daisuke F., Seto E., Denda Y., Imai Y., Okamoto K., Okamura K., Furuya M., Tani H. \& Sasai K. 2018. Outcome of limb fracture repair in rabbits: 139 cases (2007-2015). Journal of the American Veterinary Medical Association. 252(4): 457-463.

14 Shoemaker R.W. \& Wilson D.G. 2007. Surgical repair of femoral fractures in New World camelids: five cases (19962003). Australian Veterinary Journal. 85(4): 148-52.

15 Von Pfeil D.J. \& DeCamp C.E. 2009. The epiphyseal plate: physiology, anatomy, and trauma. Compendium on Continuing Education for the Practising Veterinarian. 31(8): 1-11. 